\title{
Small Bowel Metastatic Cancer Observed With Double Balloon Enteroscopy in a Patient With a Past History of Multiple Cancers
}

\author{
Ji Young Song, Beom Jae Lee, Eun Sang Yu, Young Ju Na, Jong-Jae Park, Jae Seon Kim, Young-Tae Bak \\ Division of Gastroenterology, Department of Internal Medicine, Korea University Medical Center, Seoul, Korea
}

Small bowel tumors are very rare and generally malignant. As a result of the anatomical location and nonspecific manifestations of small bowel tumors, they are very difficult to diagnose. Balloon-assisted enteroscopy is a relatively noninvasive method compared to surgical resection, and allows for real-time observation, tissue confirmation with biopsy, and interventional procedures. Here, we report the case of a 69-year-old woman with a small bowel metastatic carcinoma observed with double balloon enteroscopy (DBE). She had a history of multiple cancers including ovarian cancer, bladder cancer, and breast cancer. The antegrade DBE procedure was performed before surgery for biopsy tissue confirmation. The patient underwent small bowel resection, and the final diagnosis was the same as that determined by preoperative biopsy. The final diagnosis was metastatic small bowel cancer originating from a cancer of the breast. This is the first detailed report of the preoperative diagnosis of small intestinal metastatic breast cancer by DBE. (Intest Res 2015;13:350-354)

Key Words: Small bowel; Metastatic cancer; Double-balloon enteroscopy

\section{INTRODUCTION}

Small bowel cancers are very rare, accounting for only $3 \%-6 \%$ of all gastrointestinal neoplasms. ${ }^{1,2}$ Because small bowel cancers lack specific clinical manifestations, and the entire small intestine is not easy to access, it is very difficult to diagnose cancers in this region.

The development of balloon-assisted enteroscopy (BAE) and capsule endoscopy has initiated a new era in small bowel lesion diagnosis. BAE is a relatively noninvasive method compared to surgical resection, with a diagnostic yield of approximately $43 \%-80 \%$ for small bowel diseases. ${ }^{3}$ BAE has advantages over other diagnostic modalities such as capsule

Received August 18, 2014. Revised September 30, 2014.

Accepted October 10, 2014.

Correspondence to Beom Jae Lee, Department of Internal Medicine, Korea

University Guro Hospital, 148 Gurodong-ro, Guro-gu, Seoul 08308, Korea.

Tel: +82-2-2626-3004, Fax: +82-2-853-1943, E-mail: L85210@korea.ac.kr

Financial support: None. Conflict of interest: None. endoscopy, not only in terms of access to the entire small bowel with real-time observation, but also in facilitating endoscopic intervention, tissue confirmation with biopsy, and procedures such as stenting and balloon dilatation. ${ }^{4-6}$

Here, we report a case of small bowel metastatic carcinoma observed with double balloon enteroscopy (DBE) in a patient with a history of multiple cancers.

\section{CASE REPORT}

A 69-year-old woman was referred to our clinic complaining of cramping abdominal pain that had persisted for 10 days and had been aggravated for one day before admission due to a small bowel obstruction. She had a history of multiple cancers including ovarian cancer (treated by total abdominal hysterectomy and bilateral salpingo-oophorectomy 12 years prior), bladder cancer (treated by transurethral resection of the bladder tumor 5 years prior), and breast cancer (treated by left modified radical mastectomy followed

๑ Copyright 2015. Korean Association for the Study of Intestinal Diseases. All rights reserved.

This is an Open Access article distributed under the terms of the Creative Commons Attribution Non-Commercial License (http://creativecommons.org/licenses/by-nc/4.0)

which permits unrestricted non-commercial use, distribution, and reproduction in any medium, provided the original work is properly cited. 
by adjuvant chemotherapy 6 years prior). On admission, her blood pressure was 110/70 $\mathrm{mmHg}$, heart rate was 92 beats/ min, body temperature was $36.4^{\circ} \mathrm{C}$, and respiratory rate was 20 breaths/min. Laboratory data revealed anemia (white blood cells, $8,100 / \mathrm{mm}^{3}$; hemoglobin, $8.0 \mathrm{~g} / \mathrm{dL}$; platelets $\left.297,000 / \mathrm{mm}^{3}\right)$, and biochemical tests revealed no abnormal findings (CRP, $4.18 \mathrm{mg} / \mathrm{L}$; glucose $162 \mathrm{mg} / \mathrm{dL}$; BUN, $11.6 \mathrm{mg} /$ dL; creatinine, $0.73 \mathrm{mg} / \mathrm{dL}$; AST, $20 \mathrm{IU} / \mathrm{L}$; ALT, $13 \mathrm{IU} / \mathrm{L}$; total bilirubin, $0.48 \mathrm{mg} / \mathrm{dL}$; protein, $7.2 \mathrm{~g} / \mathrm{dL}$; albumin, $4.0 \mathrm{~g} / \mathrm{dL}$; PT, 13.3 seconds [International Normalized Ratio 1.0], and aPTT, 29.7 seconds). Urinalysis did not reveal any abnormalities.

Neither esophagogastroduodenoscopy nor colonoscopy revealed any specific findings. Conventional abdominal CT scans revealed a circumferential wall-thickening lesion in the proximal to mid jejunum, and because the patient had a history of multiple cancers, this lesion was treated as suspicious and assumed to represent metastatic small bowel cancer or (less likely) adenocarcinoma (Fig. 1A, B). A fluoro- scopic small bowel follow-through revealed a 2-cm circumferential wall-thickening lesion appearing as an "apple core sign" in the mid jejunum (Fig. 1C). For pathological confirmation, antegrade DBE (EN450T5, Fujinon, Saitama, Japan) was performed under conscious sedation. An approximately 2-cm long encircling ulcero-infiltrative lesion was noted at the mid jejunum (depth of insertion approximately $100 \mathrm{~cm}$ ) with partial luminal obstruction (Fig. 2). The total procedure time was approximately 50 minutes. Two pieces of tissue were biopsied, and histological examination revealed atypical epithelial nests with nuclear pleomorphism and frequent mitosis (Fig. 3); the biopsy result was strongly suggestive of metastatic breast cancer. The patient underwent small bowel resection for her continuing abdominal pain, and the final pathological diagnosis was small bowel metastatic carcinoma originating from the breast (Fig. 4). The patient underwent chemotherapy in the oncology department, and we administered docetaxel monotherapy, the most common drug for the treatment of breast cancer $\left(75 \mathrm{mg} / \mathrm{m}^{2}\right.$ on day
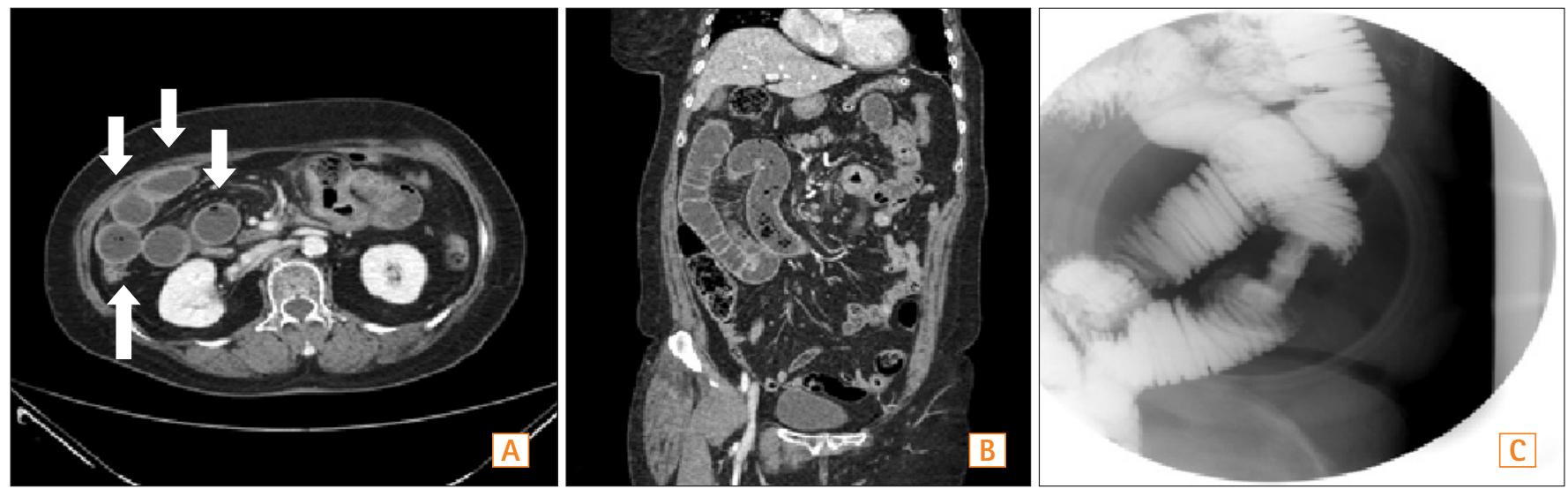

Fig. 1. Diagnostic studies. (A) Abdominal CT showing a circumferential wall-thickening lesion in the proximal to mid jejunum, which was suspected to be malignant (axial view; arrow). (B) Abdominal CT (sagittal view). (C) Small bowel follow-through showing a 2-cm circumferential wall thickening, illustrating the "apple core sign" in the mid jejunum.
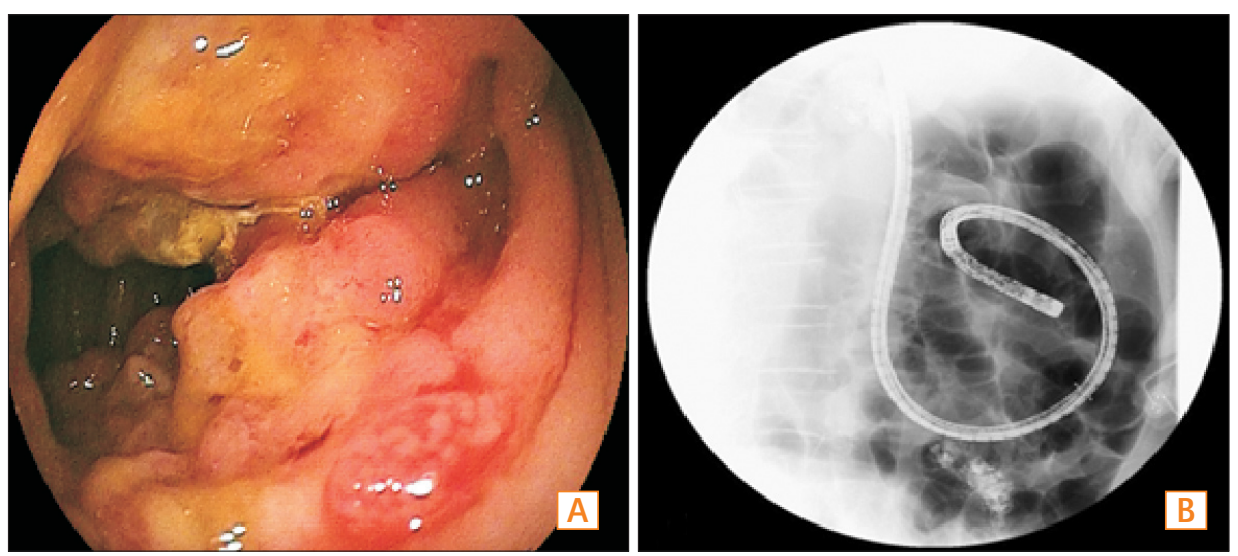

Fig. 2. Endoscopic findings. (A) Antegrade double balloon enteroscopy (DBE) showing a $2-\mathrm{cm}$ long encircling the ulceroinfiltrative lesion in the mid jejunum. (B) Fluoroscopy during DBE. 

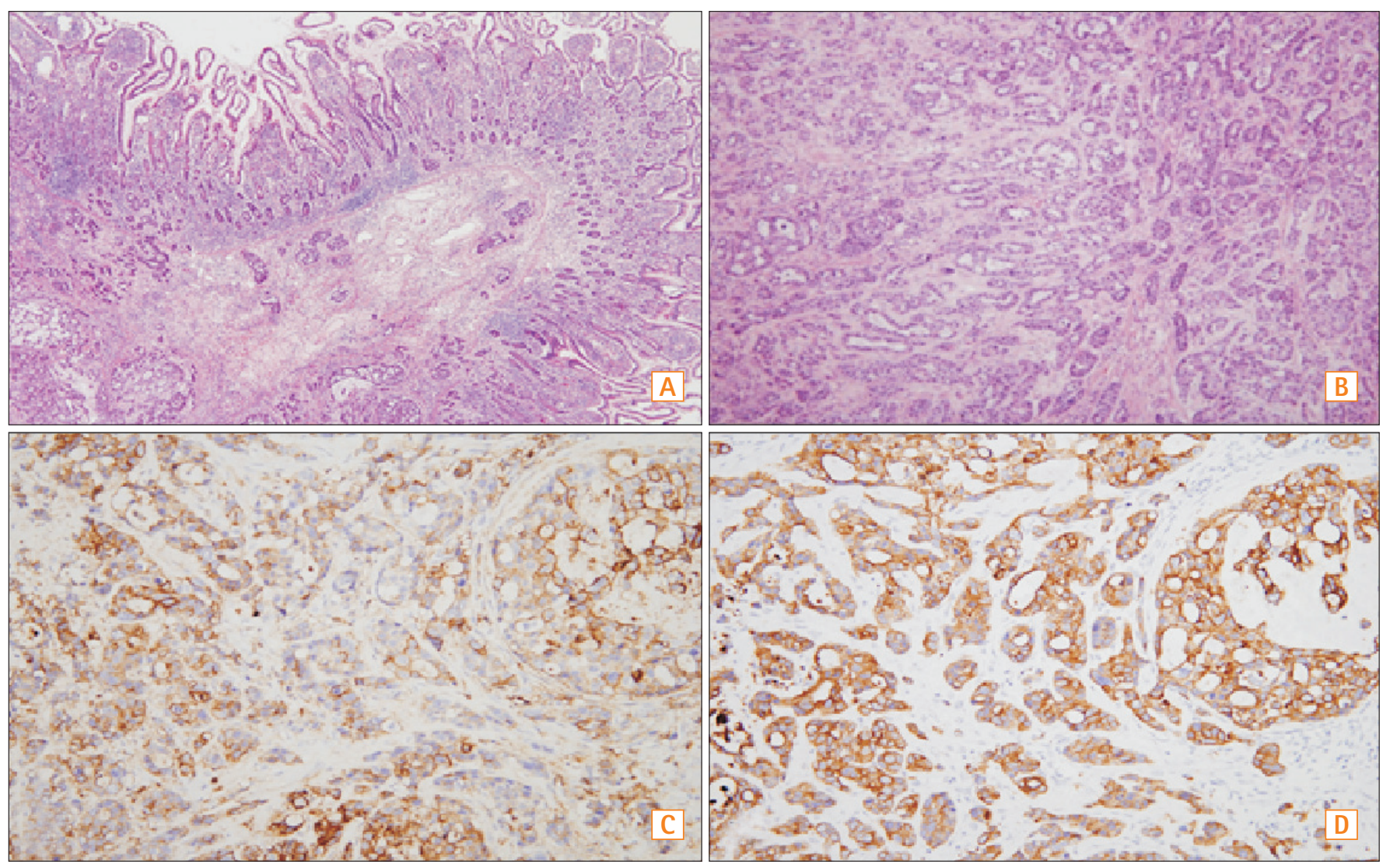

Fig. 3. Histopathologic analysis by endoscopic biopsy. (A) Many atypical epithelial nests, with nuclear pleomorphism and frequent mitosis, suggesting metastatic carcinoma from the breast $(H \& E, \times 40)$. (B) Metastatic carcinoma from the breast $(H \& E, \times 100)$. (C) Positive immunohistochemical staining for CD44. (D) Positive immunohistochemical staining for CD7.

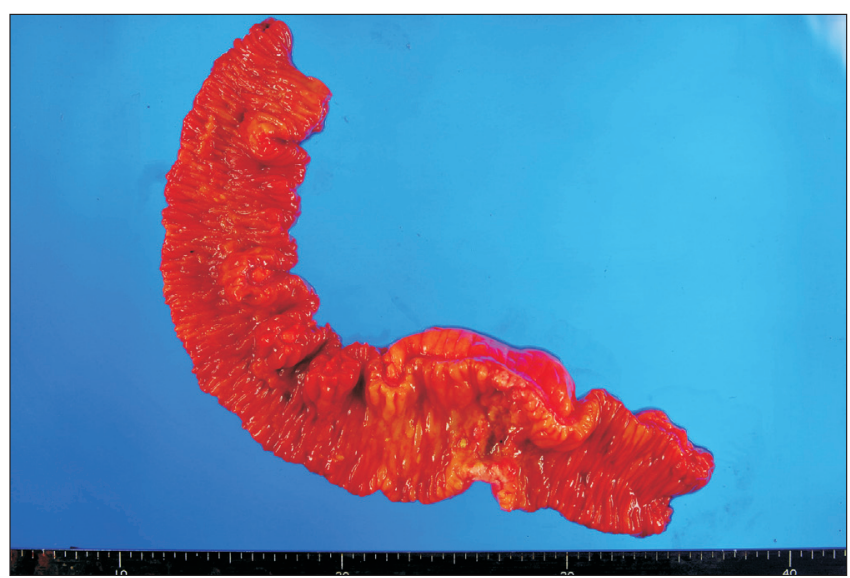

Fig. 4. Gross photography of the resected specimen of small bowel.

1, 21 days for 1 cycle). Following 6 cycles of chemotherapy, new metastatic lesions in the brain were observed, and brain radiotherapy was performed 1 year after diagnosis. Three years have passed since the initial diagnosis. No remnant lesions or new lesions have been found, and the patients vis- ited the hospital every 3 months for follow-up investigations under good conditions.

\section{DISCUSSION}

Since the introduction of DBE, small bowel tumors can be easily diagnosed without surgical resection, and several studies have reported the characteristics of diagnosing small bowel tumors using DBE. Recent studies concerning small bowel tumors using DBE have shown that the most common type of small bowel carcinoma is metastatic small bowel cancer followed by adenocarcinoma, carcinoid tumors, and lymphoma. ${ }^{1,3}$

Metastatic small bowel cancer is more frequent than primary small bowel cancer. However, the overall incidence of metastatic small bowel cancer is very low. The most common symptoms are abdominal pain, nausea, vomiting, and overt gastrointestinal tract bleeding. The most frequent endoscopic findings of small bowel cancer are stenosis or ulceration, followed by submucosal tumors and multiple 
white nodules. ${ }^{4}$ In addition, the most frequent location for small bowel tumors is the jejunum, followed by the ileum and duodenum. ${ }^{2}$

Although there are some differences between the studies reported to date, the most common metastatic cancers involving the small bowel are primary tumors such as malignant melanoma, colon cancer, cervical cancer, and ovarian cancer. $^{7}$ Metastasis to the small bowel is usually hematogenous or due to direct invasion from intraperitoneal seeding. Primary cancers of the colon, ovary, uterus, and stomach are usually spread to the small bowel by direct invasion or intraperitoneal spread, whereas primary cancers from the breast, lung, and melanoma metastasize to the small bowel by a hematogenous route. ${ }^{8}$ The overall incidence of metastatic small bowel cancer is very low, and as mentioned earlier, most metastatic cancers have neither specific symptoms nor signs until they cause small bowel obstruction. Therefore, it is very difficult to detect metastatic small bowel cancer. Due to the delays in diagnosis, patients with metastatic small bowel cancer are diagnosed at relatively advanced stages and usually have a poor prognosis. ${ }^{4}$

The present case involved small bowel obstructions of unknown origin in a patient with a history of multiple cancers. Patients with small bowel obstruction usually require surgical resection to relieve the obstructive symptoms and diagnose the etiology. However, the preoperative assessment of small bowel obstructions by DBE enables surgeons to perform a scheduled operation by confirming the preoperative pathologic findings of the cancer's etiology, and to prevent extensive small bowel resection by determining the extent or location of obstructive lesions. Moreover, in recent years, the role of BAE has expanded. Besides diagnostic evaluation, deep small bowel enteroscopy enables the widening of bowel-obstructive lesions by endoscopic guided metal stent insertion. ${ }^{9}$

Recurrence or metastasis of breast cancer is common, and occurs in $20 \%-30 \%$ of patients. The common sites for breast cancer metastasis are bone, lung, liver, and brain. ${ }^{7,10}$ Metastasis to the gastrointestinal tract is uncommon and when it does occur, it mainly involves the stomach, colon, or rectum. ${ }^{11,12}$ Metastasis to the small bowel is very rare, comprising approximately $0.08 \%$ of metastatic breast cancers. ${ }^{13}$ There are very few reports of the small intestinal metastasis of breast cancer.

In the present case, metastatic small bowel cancer was diagnosed 6 years after the initial diagnosis of breast cancer. The median interval for recurrence after surgical resection for breast cancer is 2 to 3 years, and recurrence is within 5 years in $90 \%$ of cases, although a 30 -year interval between the initial diagnosis of breast cancer and the presentation of metastasis has been reported in the literature. ${ }^{7,11}$ Therefore, metastatic small bowel cancer should be considered in patients with gastrointestinal symptoms or a newly identified gastrointestinal neoplasm, especially those with a history of colon cancer, ovarian cancer, lung cancer, or breast cancer, even if the patients were diagnosed with cancer a long time ago. $^{14}$

In this paper, we reported small bowel obstruction due to small bowel metastasis from breast cancer diagnosed by DBE in a patient with a history of multiple cancers. To our knowledge, this is the first detailed report of the preoperative diagnosis of small intestinal metastatic breast cancer by DBE. Small bowel metastatic cancer is a rare disease and not easy to detect early. There is no doubt that DBE is one of the most useful devices for approaching a suspected small bowel tumor, and for making a histological diagnosis before surgery.

\section{REFERENCES}

1. Williamson JM, Williamson RC. Small bowel tumors: pathology and management. J Med Assoc Thai 2014;97:126-137.

2. Lee BI, Choi H, Choi KY, et al. Clinical characteristics of small bowel tumors diagnosed by double-balloon endoscopy: KASID multi-center study. Dig Dis Sci 2011;56:2920-2927.

3. Chen WG, Shan GD, Zhang H, et al. Double-balloon enteroscopy in small bowel tumors: a Chinese single-center study. World J Gastroenterol 2013;19:3665-3671.

4. Imaoka H, Higaki N, Kumagi T, et al. Characteristics of small bowel tumors detected by double balloon endoscopy. Dig Dis Sci 2011;56:2366-2371.

5. Mitsui K, Tanaka S, Yamamoto H, et al. Role of double-balloon endoscopy in the diagnosis of small-bowel tumors: the first Japanese multicenter study. Gastrointest Endosc 2009;70:498504.

6. Choi MH, Lee KH, Ryu JH, et al. The usefulness of double balloon enteroscopy for diagnosis and treatment of small bowel diseases. Intest Res 2008;6:31-36.

7. Nazareno J, Taves D, Preiksaitis HG. Metastatic breast cancer to the gastrointestinal tract: a case series and review of the literature. World J Gastroenterol 2006;12:6219-6224.

8. Choi JE, Park SY, Jeon MH, et al. Solitary small bowel metastasis from breast cancer. J Breast Cancer 2011;14:69-71.

9. Park JJ, Cheon JH. Malignant small bowel obstruction: the last frontier for gastrointestinal stenting. J Gastroenterol Hepatol 2012;27:1136-1137. 
10. Eljabu W, Finch G, Nottingham J, Vaingankar N. Metastatic deposits of breast lobular carcinoma to small bowel and rectum. Int J Breast Cancer 2011;2011:413949.

11. Mouawad NJ, Cleary RK. Small bowel obstruction as the primary presentation of undiagnosed metastatic lobular breast carcinoma. Breast Dis 2011;33:35-40.

12. Lv L, Zhao Y, Liu H, Peng Z. Case report of small bowel obstruction caused by small intestinal metastasis of bilateral breast cancer. Exp Ther Med 2013;6:675-678.
13. Kawasaki A, Mimatsu K, Oida T, et al. Small intestinal perforation due to metastasis of breast carcinoma: report of a case. Surg Today 2011;41:698-700.

14. Shim JH, Son EJ, Lim BJ, Youk JH, Kim JA, Jeong J. Localized metastasis to small and large bowel from breast cancer: a case report. J Korean Soc Radiol 2010;62:551-554. 